Conclusion There was no evidence of an anti-tussive effect of GSK2339345 over the $8 \mathrm{~h}$ analysis for any subject, despite cough frequency being highly reproducible within patients. Inhalation of GSK2339345 had a pro-tussive effect in all subjects following actuation of the device, not seen with placebo. The novel cough challenge methodology warrants further investigation as a development tool.

\section{P239 LOW PREVALENCE OF EXTRA-THORACIC AIRWAY HYPER-RESPONSIVENESS IN UK PATIENTS WITH CHRONIC REFRACTORY COUGH}

${ }^{1} \mathrm{~A}$ Menon, ${ }^{2} \mathrm{~J} \mathrm{Hulll},{ }^{2} \mathrm{KF}$ Chung, ${ }^{2} \mathrm{O}$ Usmani, ${ }^{2} \mathrm{~S}$ Ward. ${ }^{1}$ Imperial College London, London, UK; ${ }^{2}$ Royal Brompton Hospital, London, UK

\subsection{6/thoraxjnl-2015-207770.375}

Introduction Prior research indicates that chronic refractory cough (CRC) is associated with a high prevalence of extra-thoracic airway hyper-responsiveness (EAHR). ${ }^{1}$ This heightened laryngoconstrictor reflex can be characterised using standard bronchoprovocation tests (e.g. histamine or hypertonic saline); whereby attenuation in the inspiratory component of the flowvolume curve is evaluated in response to escalating doses of the stimulus.

Aims and objectives To determine the prevalence of EAHR in a cohort of CRC patients in the UK undergoing cough assessment, and to relate EAHR to other disease characteristics.

Methods Data was retrospectively evaluated for all CRC patients completing cough assessment with histamine bronchoprovocation challenge, between 2013 and 2015. EAHR was defined by a $25 \%$ dose-responsive fall in the mid-inspiratory flow (PC25FIF50) in response to $\leq 8 \mathrm{mg} / \mathrm{ml}$ histamine. ${ }^{2}$ EAHR data was compared with other simultaneous investigation results, including overnight $\mathrm{pH} / \mathrm{impedance}$ results and co-existing nasal disease.

Results We studied 57 adult CRC patients ( $\mathrm{n}=42$, female; $74 \%$ ), mean \pm SD age $54.6 \pm 12.4$ years, BMI $28.2 \pm 5.9 \mathrm{~kg} /$ $\mathrm{m}^{2}$, reporting a duration of cough 5.5 years $(0.8-50)$ with a median cough VAS score of 57 (16-90). The majority of patients $(56 \%)$ reported cough without other respiratory symptoms, whereas 12 (21\%) reported cough with dyspnoea and wheeze. Evidence of EAHR was found in three patients (5.3\%). At a reduced cut-off (PC20FIF50 $\leq 16 \mathrm{mg} / \mathrm{ml}$ ) the prevalence of EAHR was greater (12\%) (Figure 1). Patients with a positive EAHR test at this cut-off were younger $(\mathrm{p}<0.01$, mean age 44 yrs versus 56 yrs) and more likely to report respiratory dyspnoea and wheeze $(\mathrm{p}<0.05)$. In patients completing an overnight reflux study $(\mathrm{n}=52), 32(62 \%)$ had evidence of reflux. $21(37 \%)$ patients had co-existing nasal disease. However, presence of reflux or nasal disease was not predictive of EAHR (both $\mathrm{p}>0.05$ ).

Conclusion EAHR was not prevalent in CRC patients, completing assessment at a specialist cough service, when using a standard histamine bronchoprovocation test. Differences from prior published data may be explained by methodological differences, specifically the application of stringent control of the measures of reproducibility of inspiratory flow parameters and dose response criteria.

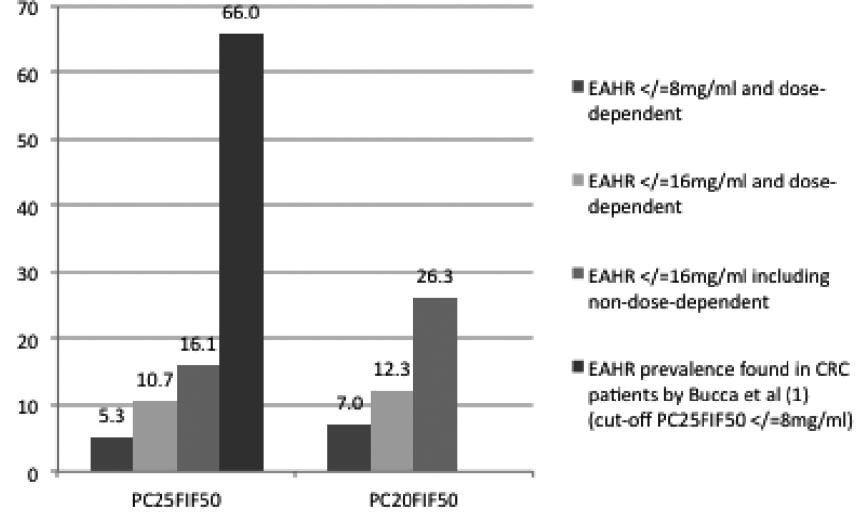

Abstract P239 Figure 1

\section{REFERENCES}

1 Bucca C, Rolla G, Scappaticci E, et al. Histamine hyperresponsiveness of the extrathoracic airway in patients with asthmatic symptoms. Allergy 1991;46: 147-53

2 Bucca C, Bugiani M, Culla B, et al. Chronic cough and irritable larynx. J Allergy Clin Immunol. 2011;127:412-9

\section{P240 VALIDATION OF THE LEICESTER COUGH QUESTIONNAIRE IN PULMONARY TUBERCULOSIS}

${ }^{1} \mathrm{RD}$ Turner, ${ }^{1} \mathrm{GH}$ Bothamley, ${ }^{2} \mathrm{SS}$ Birring. ${ }^{1}$ Homerton University Hospital NHS Foundation

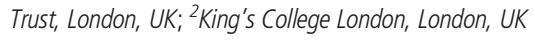

\subsection{6/thoraxjnl-2015-207770.376}

Introduction and objective Cough is prominent in pulmonary tuberculosis (TB) and transmits infection, yet no tool has been validated for assessing cough symptoms. We evaluated the Leicester Cough Questionnaire (LCQ) for measuring cough-related quality of life (QOL) in TB.

Method The face validity of the LCQ was evaluated by structured interviews with patients and a multi-disciplinary team (MDT) discussion (respiratory physicians and nurses). Consecutive patients with TB completed the LCQ just before or within 7 days of starting therapy; a subgroup completed a repeat questionnaire approximately two weeks after the first. Internal reliability (inter-relatedness between items), concurrent validity (association with cough severity visual analogue scale [VAS] score and 24-hour cough frequency measured with the Leicester Cough Monitor), and responsiveness were evaluated.

Results The MDT and patients thought the LCQ to be relevant, comprehensive and useful in TB and no modifications were suggested. Forty patients completed the questionnaire before $(\mathrm{n}=$ $29)$ or just after $(n=11)$ the start of treatment. Internal reliability of responses was high (Cronbach's $\alpha=0.93$ ). LCQ scores were correlated with both the VAS (Spearman's $\rho=-0.69[95 \%$ confidence intervaI -0.83 to -0.46$], p<00.0001)$ and 24 -hour cough frequency ( $\rho=-0.36[-0.62$ to -0.04$], p=0.023$ ), and were worse pre-treatment in culture-positive compared to culture-negative disease (median 12.4 [IQR 8.5-17.4] vs 18.7 [17.8-19.6] respectively, $p=0.052$ ). There was no evidence of association with other markers of disease severity (sputum smear positivity, lung cavities and radiographic extent of disease), but a trend towards worse LCQ scores amongst current smokers than non-smokers (12.6 [8.3-14.4] vs 17.1 [11.1-21.0] respectively, $p$ $=0.075)$. 
All patients who repeated the questionnaire appeared adherent to TB medication. There were substantial improvements in LCQ responses after a median of $14(10-14)$ days' treatment ( $\mathrm{n}=12$; median [IQR] score 9.1 [8.1-14.5] at baseline, 18.3 [14.5-19.4] at two weeks, median improvement 5.1 [1.8-9.7], $p=0.003$; Figure 1). The effect size of the change in LCQ scores was 1.17 .

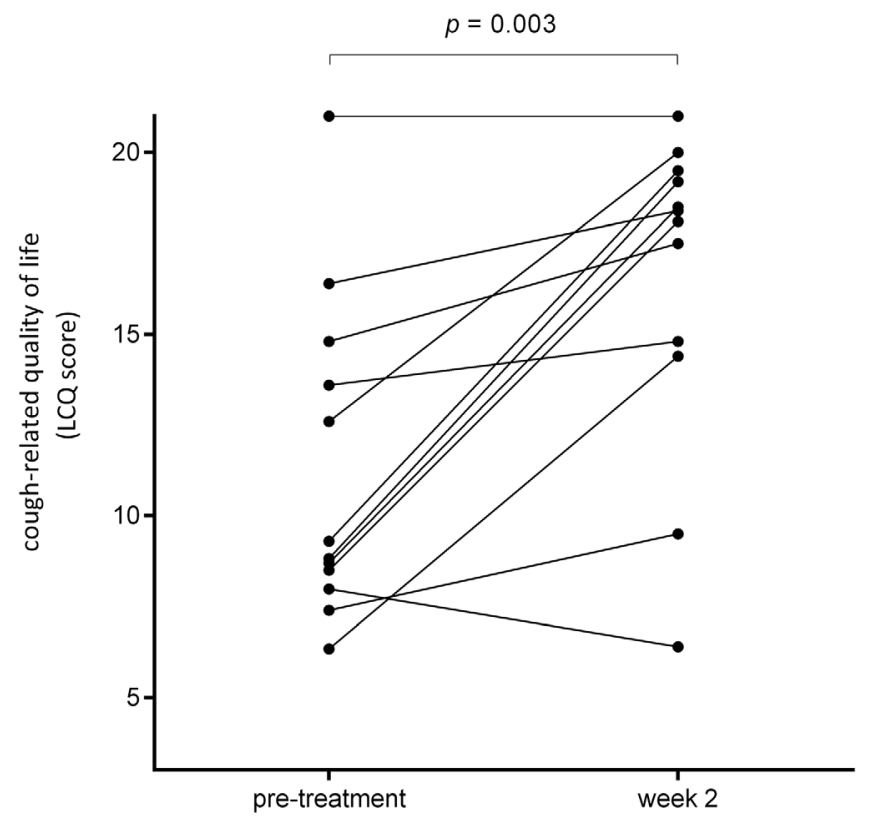

Abstract P240 Figure 1 Changes Leicester Cough Questionnaire score during early treatment of pulmonary tuberculosis

Conclusion The LCQ is a valid instrument for evaluating coughrelated QOL in TB and may be a useful outcome measure to evaluate therapy.

\section{P241 THE FEASIBILITY AND VALIDITY OF OBJECTIVE COUGH MONITORING IN CHILDREN USING AN ADULT COUGH DETECTION SYSTEM}

D Deblej Elghamoudi, H Sumner, K McGuiness, J Smith, CS Murray. University of Manchester, Manchester, UK

\subsection{6/thoraxjnl-2015-207770.377}

Background In young children the evaluation of symptoms is almost wholly reliant upon parental reporting. Cough is extremely common in childhood and a tool to objectively measure cough frequency would be clinically beneficial. To date paediatric cough monitoring systems have relied on manual cough counting which is time consuming and costly. The VitaloJAK $^{\mathrm{TM}}$ (Vitalograph, UK) is a custom built $24 \mathrm{hr}$ semi-automated cough monitoring device that has been used successfully to quantify cough in adults. Using the VitaloJAK ${ }^{\mathrm{TM}}$ we tested the feasibility of $24 \mathrm{hr}$ cough recordings in children and tested existing compression software for cough quantification.

Methods Children (age 2-14 years) with acute or chronic cough were asked to wear VitaloJAK ${ }^{\mathrm{TM}}$ cough monitor for a maximum of $24 \mathrm{~h}$. Feedback about the device was obtained from the child/ carer were appropriate. All recordings were manually counted and also processed through the compression software.
Results 40 children ( 21 male; mean age 8 yrs) wore the monitor for a median of $22.25 \mathrm{~h}(0.38-24 \mathrm{hrs})$. Children who wore the monitor as out-patients with chronic cough generally wore the monitor for longer (median $23.07 \mathrm{hrs}$ ) than those who were in-patients with acute symptoms (median $12.59 \mathrm{hrs;} \mathrm{p}=0.06$ ). Twenty-nine children (73\%) wore the monitor for $>12 \mathrm{~h}$ and 22 for $>22$ hrs including during the night. Eighteen (45\%) children reported the monitor was heavy/bulky and eleven (28\%) said at times it restricted some normal daily tasks (bending/stooping/ using the toilet). Neither length of time the monitor was worn for, nor complaints about the size or restricting nature of the monitor, were age dependent. No serious adverse events were reported. One recording was excluded due to technical problems. Recordings demonstrated wide variability in frequency from 2 to 2712 coughs per recording (median 72). Following compression a median of $98.5 \%(85.7-100)$ of coughs were retained.

Conclusions VitaloJAK ${ }^{\mathrm{TM}}$ semi-automated cough monitor can be used in children to accurately measure cough frequency. As with many medical interventions, it was not tolerated by all children for long periods, however the majority of children were able to wear it for $>12 \mathrm{~h}$.

\section{P242 THE ORDER EFFECT OF EXPERIMENTAL OESOPHAGEAL ACIDIFICATION ON COUGH REFLEX SENSITIVITY IN CHRONIC COUGH PATIENTS AND HEALTHY VOLUNTEERS}

D Valdramidou, H Sumner, E Hilton, S Whiteside, JA Smith. University of Manchester/ University Hospital South Manchester NHS, Manchester, UK

\subsection{6/thoraxjnl-2015-207770.378}

Background One of the mechanisms thought to be involved in the triggering of chronic cough is gastroesophageal reflux. We aimed to compare the effects of oesophageal acid infusion on cough reflex sensitivity in chronic cough (CC) patients with healthy volunteers (HV).

Method In a randomised, double-blind, placebo-controlled, crossover study, $0.15 \mathrm{M}$ hydrochloric acid/saline was infused in the distal oesophagus of each subject. Each infusion treatment was separated by a washout period of at least 7 days. Single dose inhalation capsaicin challenge $\left(\mathrm{C}_{5}\right)$ was used to measure cough reflex sensitivity before (baseline), immediately after $(\mathrm{t}=$ 0 mins) and $90 \mathrm{~min}, 180 \mathrm{~min}$ and $24 \mathrm{hrs}$ post infusion. Data were analysed using Generalised Estimating Equation (GEE) models.

Results Ten CC patients (mean age $56.0 \pm 11.0$ years, 8 females) and twelve HV (mean age $26 \pm 8.3$ years, 7 females) completed the study. Overall, CC patients had a more sensitive cough reflex than HV (geometric mean ratio 0.46; ratio 0.05, Table 1). Furthermore, there was a significant order effect (acid-saline vs saline-acid); CC patients appeared to be sensitised to acid infusion when given first $(\mathrm{p}$-value $=0.039)$, but not second $(\mathrm{p}$-value $=$ 0.245), whereas HV cough responses were not influenced by the infusion order (all p-values $>0.05$ ).

Conclusions In our experience, distal oesophageal acid infusion did not sensitise the cough reflex in either CC patients or HV. Notably, the order of the infusions heavily influenced the cough responses of CC patients compared to HV. Future study designs need to be cognisant of order effects and if cross-over designs are used, test for the presence of these. 